\title{
Croissance de juvéniles de Nereis diversicolor nourris avec des détritus d'halophytes
}

\section{Growth of Nereis diversicolor (L.) juveniles fed with detritus of halophytes}

\author{
Tarik Meziane *, Christian Retiere \\ Laboratoire Maritime de Dinard, Muséum National d'Histoire Naturelle BP 28, Dinard 35801, France
}

Reçu le 11 décembre 2001; reçu en forme révisée le 26 février 2002; accepté le 12 mars 2002

\begin{abstract}
Résumé
L'effet comparé de la prise de détritus frais et dégradés de phanérogames halophiles récoltés dans les marais salés de la baie du Mont Saint-Michel (France), sur la croissance d'une population juvénile de l'annélide polychète Nereis diversicolor a été étudié en milieu expérimental durant l'été 1993. Les détritus frais et dégradés de Spartina anglica, Halimione portulacoides et Salicornia europeae,en plus de l'algue verte Enteromorpha sp., ont été fournis séparément à des lots homogènes de juvéniles pendant une durée de 75 jours. Tous lots confondus, le taux de croissance le plus élevé a été obtenu avec les vers nourris avec Enteromorpha sp. Par ailleurs, pour une même espèce d'halophyte, la nutrition de juvéniles de $N$. diversicolor avec des détritus frais permet toujours une croissance significativement plus élevée qu'avec les détritus dégradés. Cette différence est probablement due à une biomasse microbienne élevée présente sur les aliments frais et qui, de plus, est bien mieux assimilée que les détritus qu'elle colonise. () 2002 Ifremer/CNRS/IRD/Éditions scientifiques et médicales Elsevier SAS. Tous droits réservés.
\end{abstract}

\begin{abstract}
The comparative effect of the uptake of fresh and degraded detritus of halophytic plants, harvested from salt marches of the Mont Saint-Michel Bay (France), on the growth of a juvenile population of the annelid polychaete N. diversicolor (L.) was studied under experimental conditions in summer 1993. Fresh and degraded detritus of Spartina anglica, Halimione portulacoides and Salicornia europeae, as well as the green algae Enteromorpha sp. were distributed separately to homogenous set of juveniles for a $75 \mathrm{~d}$ period. The highest growth rate was obtained for worms fed with Enteromorpha sp. Administration of fresh detritus of a given halophyte species to $\boldsymbol{N}$. diversicolor juveniles always leads to a significantly higher growth rate than did degraded detritus. This is probably due to a great microbial biomass occurring on the fresh detritus, which is moreover, much better assimilated than the detritus they colonize. (C) 2002 Ifremer/CNRS/IRD/Éditions scientifiques et médicales Elsevier SAS. All rights reserved.
\end{abstract}

Mots clés: Nereis diversicolor; Croissance; Halophytes; Détritus

Keywords: Nereis diversicolor; Growth; Halophytes; Detritus

\section{Introduction}

En système côtier, les fonds de baies soumis à l'alternance des marées, au même titre que les estuaires, sont un lieu privilégié de transferts de matière organique provenant

\footnotetext{
* Auteur correspondant : Laboratory of Ecology and Systematics, Faculty of Science, University of the Ryukyus, Senbaru 1, Nishihara 903-0213, Okinawa, Japan.

Adresse e-mail : h983001@sci.u-ryukyu.ac.jp (T. Meziane).
}

aussi bien d'apports continentaux et marins que des productions autochtones. Parmi celles-ci, la végétation halophile des marais salés constitue l'un des systèmes végétaux les plus productifs au monde et intervient pour une très grande part dans la production primaire totale de la zone intertidale (Gordon et al., 1985 ; Vernberg, 1993 ; Mitsch et Gosselink, 1993). Ces marais salés produisent beaucoup plus de matériel organique qu'il n'en est utilisé, dégradé ou stocké sur place (Teal, 1962 ; Odum, 1980). Cet excès de matériel 
organique est exporté à différents stades de dégradation vers les estrans adjacents (Valiela et Teal, 1979 ; Nixon, 1980) et intègre le réseau trophique (Schwinghamer et al., 1986 ; Meziane et al., 1997 ; Kneib et al., 1997). Cependant, on ignore si, en milieu naturel, les détritus d'halophytes sont consommés par les macro-invertébrés préférentiellement sous une forme fraîche ou plus au moins dégradée.

Le rôle des détritus d'halophytes dans la croissance des invertébrés marins est désormais reconnu (Tenore, 1977, 1981, 1983 ; Lopez et Levinton, 1987 ; Garça et al., 2000). Cependant, en raison du caractère réfractaire de la structure ligno-cellulosique de ces phanérogames, la matière organique d'origine halophile est moins bien assimilée que les algues chez quelques espèces de polychètes (ex : Capitella capitata, Tenore, 1977 ; Nereis virens, Olivier et al., 1996a ; $N$. diversicolor; Olivier et al., 1996b). Bactéries hétérotrophes et champignons ascomycètes jouent un rôle prédominant dans les processus de dégradation qui affectent les plantes vasculaires aquatiques (Moran et Hodson, 1989; Newel, 1993 ; Samiaji et Bärlocher, 1996). Cette dégradation a pour effet de changer la structure des plantes. Ainsi, l'activité bactérienne entraîne t-elle une augmentation des quantités d'azote (Tenore, 1981, 1983 ; Findlay et Tenore, 1982) ce qui rendrait les détritus d'halophytes potentiellement plus attractifs pour les détritivores. De plus, il est très probable que l'ingestion de la matière organique détritique par les macro-invertébrés reste le moyen le plus efficace d'accéder à la biomasse microbienne.

Le long des côtes de la Manche, l'annélide polychète $N$. diversicolor est un membre dominant de la communauté à Macoma balthica, dont la distribution est limitée, comme c'est le cas en baie du Mont Saint-Michel, à une large section de la partie la plus haute de l'estran $(400 \mathrm{~m}$, Meziane et Retière, 2001). Les juvéniles sont surtout présents sur les 200 premiers mètres. Cette espèce se caractérise par la plasticité de son comportement alimentaire; elle se comporte, lors de ses différents stades de développement, aussi bien en carnivore (Marty et al., 1997) qu'en filtreur (Vedel et Riisgard, 1993) ou qu'en déposivore de surface (Olivier et al., 1995).

En baie du Mont Saint-Michel, la flore des zones pionnières de schorre est dominée par les halophytes Salicornia spp. et Spartina anglica (Guillon, 1984). Dans la partie médiane du schorre, Halimione portulacoides est l'espèce halophile dominante. Zones pionnière et médiane des marais salés sont régulièrement inondées à marée haute, pouvant alors exporter de la matière organique particulaire.

Dans une précédente étude, des détritus dégradés des halophytes Salicornia europeae, H. portulacoides, Spartina anglica, ainsi que l'algue verte Enteromorpha intestinalis, ont été présentés comme nourriture à des juvéniles de $N$. diversicolor (Olivier et al., 1996a). La présence en abondance de détritus frais à côté des détritus à différents niveaux de dégradation sur l'estran (Meziane et Retière, obs. pers.), nous a décidé à renouveler cette étude, avec pour objectif de tester, en plus de l'influence de la nature (espèce) des halophytes, l'effet de l'état (frais ou dégradé) de ces détritus sur la croissance d'individus juvéniles de $N$. diversicolor.

\section{Matériels et méthodes}

\subsection{Récolte et préparation des détritus}

Les macrophytes qui ont été utilisées pour la préparation des détritus aussi bien frais que dégradés sont les plantes halophiles Spartina anglica, H. portulacoides et Salicornia europeae et l'algue verte Enteromorpha sp., uniquement à l'état frais. Les halophytes ont été récoltées pendant toute la durée de l'expérience (de juin à août 1993) sur le marais salé du Vivier-sur-Mer en baie du Mont Saint-Michel. L'entéromorphe a été collectée sur les substrats rocheux situés en contre bas du Laboratoire maritime de Dinard. Le protocole de dégradation des détritus consiste à placer des feuilles et des tiges de chaque plante halophile dans des récipients contenant de l'eau de mer (continuellement renouvelée), eux-mêmes maintenus à une température de $50{ }^{\circ} \mathrm{C}$ pendant un mois.

\subsection{Origine des animaux}

Les juvéniles de l'espèce $N$. diversicolor, au nombre de 1440, ont été ramassés dans la partie la plus haute de l'estran de La Richardais à l'embouchure de l'estuaire de la Rance juste en amont de l'usine marémotrice en mai 1993. De retour au laboratoire, ces vers ont été mis à jeûner pendant 24 heures afin de vider leur tube digestif avant d'être pesés individuellement (précision $\pm 0,1 \mathrm{mg}$ ).

\subsection{Protocole expérimental}

En début d'expérience, les vers ont été répartis au hasard par lot de dix dans cent quarante-quatre pots $(10 \mathrm{~cm}$ de diamètre et $15 \mathrm{~cm}$ de hauteur) contenant, jusqu'à environ $2 \mathrm{~cm}$ du bord, du sédiment vaseux préalablement défauné et tamisé sur une toile métallique de $0,5 \mathrm{~mm}$ de vide de maille. Ces pots ont été ensuite recouverts par un couvercle constitué d'une maille en plastique de $1 \mathrm{~mm}$ de diamètre dans le dessein d'empêcher une migration des individus, puis placés par groupe de dix-huit dans huit bacs $(1 ; 0,5$; $0,5 \mathrm{~m}$ ) installés dans un bassin rempli et continuellement alimenté d'eau de mer courante. Durant la durée de l'expérience, la température de l'eau a varié de $15{ }^{\circ} \mathrm{C}$ en juin à $21{ }^{\circ} \mathrm{C}$ en août ; la durée journalière de l'éclairement naturel est demeurée comprise entre 13 heures et 14 heures 30 .

Mis à part le bac témoin (bac 1), les individus de chaque bac ont été nourris avec un seul type d'aliment : S. anglica fraîche (2) et dégradée (3), H. portulacoides fraîche (4) et dégradée (5) et $S$. europeae fraîche (6) et dégradée (7) et l'algue verte Enteromorpha sp. uniquement à l'état frais (8). La présence d'un seul type d'aliment par bac pouvait 
conduire à un problème de pseudo-réplication puisque un «effet bac » éventuel était susceptible de se superposer à celui de la nourriture. Ce protocole a néanmoins été retenu car : (1) il existe une possibilité de contamination chimique par les halophytes et (2) de nombreux auteurs ont déjà adopté des plans expérimentaux similaires (Olivier et al., 1996a, b).

Tous les deux jours, $500 \mathrm{mg}$ poids frais (pf) d'aliments, préalablement découpés en petits morceaux de 1 à $2 \mathrm{~mm}$ de long, ont été déposés dans chaque pot pendant les deux mois et demi de l'expérience (ration alimentaire de $25 \mathrm{mg}$ pf $\mathrm{j}^{-1}$ ind $^{-1}$ ). Toutes les deux semaines, les individus de trois pots choisis au hasard dans chaque bac, ont été pesés (pf $\pm 0,1 \mathrm{mg}$ ) puis retirés de l'élevage.

\subsection{Analyse statistique}

Un premier traitement statistique a consisté à comparer les poids frais moyens initiaux des individus à l'intérieur de chaque pot et de chaque traitement (nourriture). Ceci a été réalisé à l'aide d'une analyse de variance à deux facteurs contrôlés faisant intervenir à la fois le «pot» et la «nourriture » comme variables indépendantes.

La démarche adoptée pour déterminer le seuil de signification des résultats expérimentaux a comporté trois étapes. La première a consisté à examiner l'effet de la durée d'élevage sur le poids frais des juvéniles de $N$. diversicolor par le biais d'analyses de variance à un facteur contrôlé réalisées individuellement pour chaque traitement. La deuxième a consisté à cerner l'effet de la nature de la plante fournie. Elle a été conduite à partir d'analyses de variance à deux facteurs contrôlés (ex: la durée d'élevage et la nourriture) réalisées d'une part sur les traitements faisant intervenir les phanérogames fraîches et d'autre part sur les traitements faisant intervenir les détritus dégradés. Enfin, la dernière étape a consisté à examiner l'effet de l'état (frais ou dégradé) de la plante offerte à partir d'analyses de variance à deux facteurs contrôlés (la durée d'élevage et l'état de la nourriture) réalisées pour chacune des plantes halophiles.

La croissance pondérale spécifique $(\mu)$ a été calculée à partir de la formule faisant intervenir le poids initial (PI), le poids final (PFi) et la durée $(t)$ de l'expérience $(\mu=((\ln (\mathrm{PFi})-\ln (\mathrm{PI})) / t)$.

\section{Résultats}

L'analyse de variance réalisée en début d'expérience n'a permis de mettre en évidence aucun effet significatif des pots et des traitements sur le poids moyen des individus utilisés (Anova à deux facteurs contrôlés, $P>0,85$ et $P>$ 0,12 , respectivement). En d'autres termes, les différents traitements ont effectivement été appliqués à des lots d'individus qui présentaient initialement des poids homogènes.

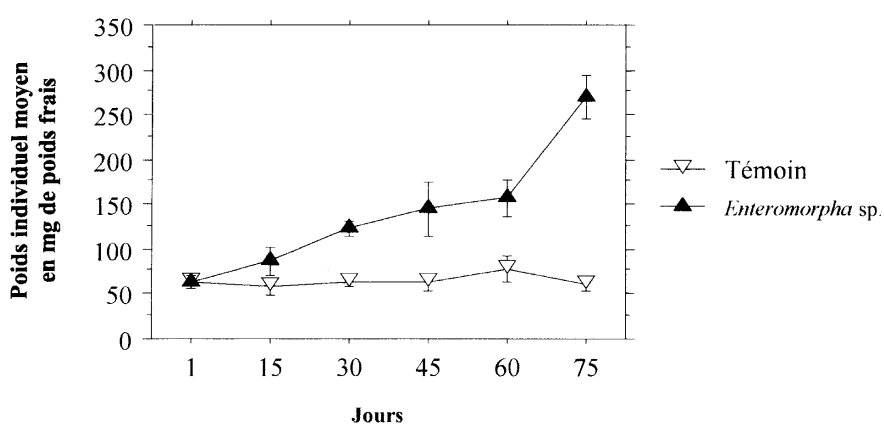

Fig. 1. Croissance pondérale (moyenne \pm écart type) des juvéniles de N. diversicolor dans les bacs «témoins » et « Enteromorpha sp. ».

Les poids moyens finaux des individus soumis au traitement « témoin » (pas de nourriture fournie) semblent légèrement inférieurs aux valeurs initiales (PI : 62,9 $\pm 4,1 \mathrm{mg}$ de poids frais contre PFi : $52,5 \pm 8,4 \mathrm{mg}$ de pf). Toutefois cette diminution n'est pas statistiquement significative (analyse de variance à un facteur contrôlé, $P>0,05$; Fig. 1 ). Ceci est contraire à ce qui est observé pour tous les autres traitements durant lesquels les poids individuels moyens augmentent toujours significativement avec la durée d'élevage (analyses de variance à un facteur contrôlé, p toujours inférieur à 0,02 ; Fig. 2).

L'augmentation des poids moyens au cours du temps n'est pas significativement affectée par la nature des phanérogames (i.e. espèce), qu'elles soient offertes à l'état frais (analyse de variance à deux facteurs contrôlés, $P>0,2$ ) ou à l'état dégradé (analyse de variance à deux facteurs contrôlés, $P>0,05)$.

Par contre, l'évolution des poids frais est significativement affectée par l'état (frais ou dégradé) de chacune des trois halophytes (analyses de variance à deux facteurs contrôlés ; $P<0,001$ pour Spartina anglica, $P<0,03$ pour $H$. portulacoides, et $P<0,02$ pour S. europeae). Dans ces trois cas, les taux de croissance des juvéniles $N$. diversicolor les plus élevés sont toujours observés chez les individus nourris avec des détritus frais (Tableau 1).

Par ailleurs, les taux de croissance des vers alimentés avec des détritus frais ou dégradés de phanérogames sont toujours inférieurs à celle des individus alimentés avec des détritus frais d'Enteromorpha sp. (seule macroalgue utilisée lors de la présente étude; Tableau 1).

\section{Discussion}

La consommation prolongée de détritus frais et dégradé, dérivés des trois plantes halophiles, S. anglica, H. portula coides et $S$. europeae, induit des croissances significatives des juvéniles de $N$. diversicolor. Toutefois, la croissance pondérale des individus alimentés avec les détritus frais n'est pas significativement différente selon que les vers ont été nourris avec l'une ou l'autre des trois espèces d'halophytes. De la même façon, l'alimentation des juvéniles avec des détritus dégradés de ces halophytes induit des 
(a)

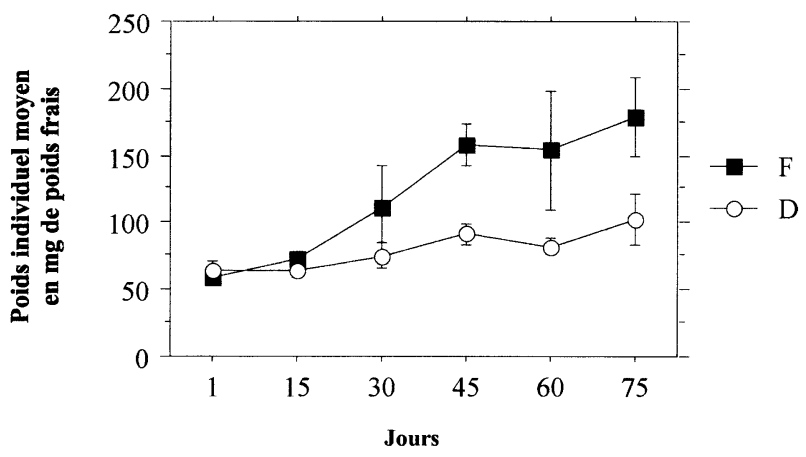

(b)

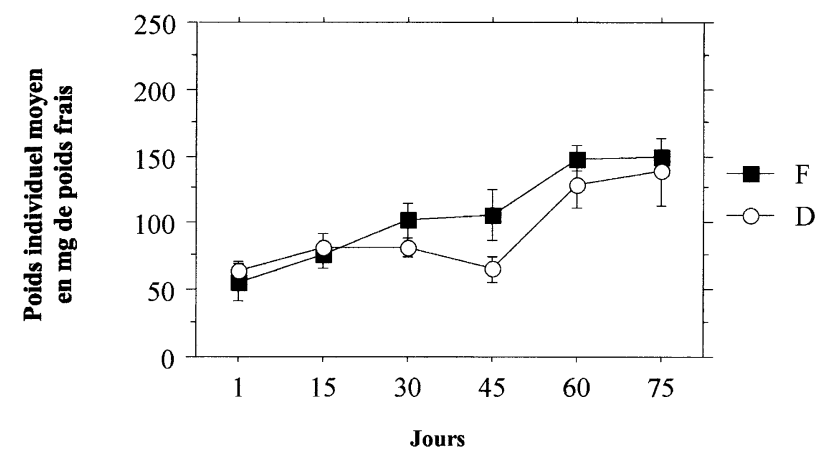

(c)

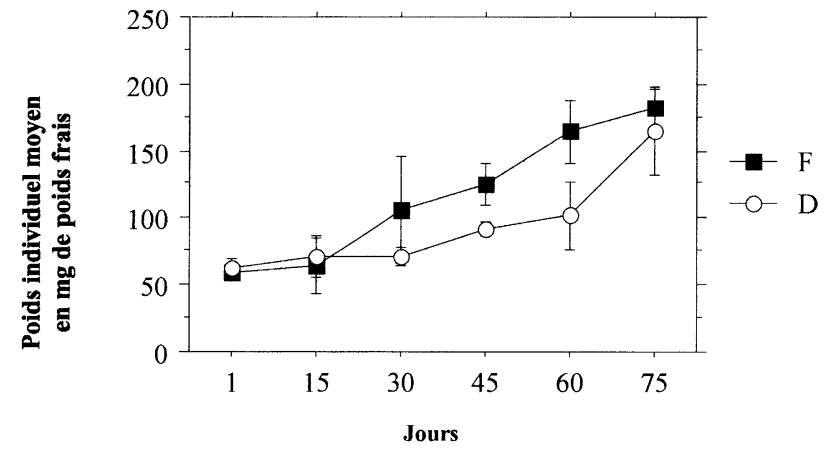

Fig. 2. Croissance pondérale (moyenne \pm écart type) des juvéniles de $N$. diversicolor alimentés avec des détritus de plantes halophiles à l'état frais (F) et dégradé (D). Plantes halophiles: (a) Spartina anglica, (b) H. portulacoides, (c) Salicornia europeae.

croissances pondérales non significativement différentes selon les espèces de plantes. Ceci est probablement lié à la grande homogénéité biochimique de ces trois phanérogames. Ces dernières ne présentent d'ailleurs pas de caractéristiques nutritives ou énergétiques propres qui pourraient induire des niveaux de croissance différents (Tableau 1 dans Olivier, 1996b). En particulier, ce résultat suggère aussi que la méthode de dégradation employée (incubation à $50^{\circ} \mathrm{C}$ ) n'entraîne pas des niveaux de dégradation différents pour les trois espèces de phanérogames utilisées.

Les détritus d'halophytes dégradés ont probablement subi une forte perte de calories disponibles qui pourrait être à l'origine de la faible croissance des juvéniles de $N$. diversicolor comparé à celle de vers alimentés avec des détritus d'halophytes frais (Tableau 1). Les taux de croissance pondérale des juvéniles de $N$. diversicolor de cette étude lorsque les individus sont nourris avec des détritus dégradés $\left(0,63\right.$ à $\left.1,30 \mathrm{j}^{-1}\right)$ sont du même ordre que ceux qui ont été mesurés précédemment, avec la même espèce, par Olivier et al. (1996b) $\left(0,56\right.$ à $\left.1,12 \mathrm{j}^{-1}\right)$ alors que les méthodes utilisées pour la dégradation des halophytes sont différentes, Olivier et al. (1996b) ayant opté pour une méthode de dégradation moins agressive $\left(12{ }^{\circ} \mathrm{C}\right.$ pendant un mois en eau de mer courante).

Pour chacune des halophytes, la croissance des juvéniles de $N$. diversicolor est toujours plus élevée pour les détritus frais que pour les détritus dégradés. En effet, tous ces taux cités plus haut sont inférieurs à ceux qui sont obtenus lorsque les annélides sont nourries avec des détritus frais $\left(1,26\right.$ à $1,50 \mathrm{j}^{-1}$, Tableau 1$)$. Ces résultats semblent en contradiction avec ceux de travaux précédents et qui montrent que les détritivores benthiques préfèrent les détritus de phanérogames dégradés aux détritus frais (Tenore, 1977, 1981 ; Tsuchiya et Kurihara, 1979). En effet, en raison de l'activité bactérienne, la dégradation de détritus frais, bien qu'elle entraîne une diminution du contenu énergétique total (Tenore, 1981), augmente la quantité de calories « disponibles » et d'azote (Tenore, 1981, 1983 ; Findlay et Tenore, 1982 ; Rice, 1982). À cet égard, Tenore (1981) indique que la croissance du polychète $C$. capitata dépend du contenu en calories et en azote des végétaux. Toutefois, cet auteur souligne à cette occasion que les contributions énergétiques, en calories disponibles, sont plus faibles dans des détritus de Spartina alterniflora ayant subi une décomposition de 2, 9 et 12 mois dans des bacs remplis d'eau de mer filtrée en circuit ouvert, que dans du matériel halophytique frais.

Durant toute la durée de l'expérience, les juvéniles de $N$. diversicolor ont régulièrement procédé à l'enfouissement de détritus frais à l'entrée de leurs galeries (obs. pers.). Ce comportement tend à montrer l'existence d'un délai entre le moment où les détritus sont mis à la disposition des vers et le moment où ces derniers les consomment laissant ainsi le temps aux bactéries et champignons de coloniser les détritus. Ce processus dit de «gardening », indépendamment du niveau de décomposition des détritus, a déjà été relevé chez $N$. diversicolor (Olivier et al., 1995). Il se pourrait alors que les juvéniles de $N$. diversicolor préfèrent se nourrir de ces détritus en voie de dégradation obtenus ainsi. En milieu naturel, les bactéries sont l'une des principales sources d'alimentation de $N$. diversicolor en baie du Mont SaintMichel (Meziane et al., 1997). Sans qu'il soit clairement établi que les bactéries se sont fixées en nombre sur les détritus frais des halophytes de cette étude, il est probable que les vers s'intéressent à ces détritus car ces derniers, par leur rôle de "support alimentaire », rendraient plus accessibles les microorganismes aux juvéniles de $N$. diversicolor, sans pour autant que les processus de dégradation microbienne interviennent pour améliorer le contenu nutritif des détritus d'halophytes. 
Tableau 1

Résultats des expériences de croissance, poids frais initial (PI) et final (PFi) (moyenne \pm écart type) et mesure du taux de croissance journalier $((\mathrm{PFi}-\mathrm{PI}) / t$ ) et de la croissance pondérale spécifique $(\mu)$

\begin{tabular}{|c|c|c|c|c|c|}
\hline Ressources & $\begin{array}{l}\text { Poids initial (PI) } \\
\text { (mg de pf) }\end{array}$ & $\begin{array}{l}\text { Poids final (PFi) } \\
\text { (mg de pf) }\end{array}$ & $\begin{array}{l}\text { Durée de l'expérience } \\
\text { (Jours) (j) }\end{array}$ & $\begin{array}{l}\text { Taux de croissance } \\
\text { journalier }\left(\mathrm{mg} \mathrm{pf}^{-1}\right)\end{array}$ & $\begin{array}{c}\text { Croissance pondérale } \\
\text { spécifique : } \mu^{*} 100 \\
\left(\% \mathrm{j}^{-1}\right)\end{array}$ \\
\hline Témoin & $62.9 \pm 4.1$ & $52.5 \pm 8.4$ & 75 & $-0.14 \pm 0.06$ & -0.24 \\
\hline Spartina anglica $\mathrm{F} *$ & $58.5 \pm 3.8$ & $178.7 \pm 23.9$ & 75 & $1.60 \pm 0.28$ & 1.49 \\
\hline Spartina anglica $\mathrm{D} *$ & $64.2 \pm 6.5$ & $103.2 \pm 15.6$ & 75 & $0.52 \pm 0.12$ & 0.63 \\
\hline Spartina anglica $\mathrm{D}^{\text {a }}$ & $48.3 \pm 12.9$ & $78.7 \pm 9.0$ & 90 & 0.36 & 0.56 \\
\hline Halimione portulacoides $\mathrm{F}$ & $58.2 \pm 5.6$ & $149.2 \pm 4.2$ & 75 & $1.21 \pm 0.019$ & 1.26 \\
\hline Halimione portulacoides D & $64.9 \pm 6.0$ & $138.7 \pm 20.7$ & 75 & $0.98 \pm 0.196$ & 1.01 \\
\hline Halimione portulacoides $\mathrm{D}^{\mathrm{a}}$ & $50.0 \pm 5.9$ & $137.6 \pm 13.5$ & 90 & 1.04 & 1.12 \\
\hline Salicornia europeae F & $59.4 \pm 3.8$ & $182.4 \pm 11.0$ & 75 & $1.64 \pm 0.096$ & 1.50 \\
\hline Salicornia europeae D & $62.3 \pm 6.8$ & $165.0 \pm 26.5$ & 75 & $1.37 \pm 0.263$ & 1.30 \\
\hline Salicornia europeae $\mathrm{D}^{\mathrm{a}}$ & $52.8 \pm 9.3$ & $132.6 \pm 6.1$ & 90 & $1.03 \pm 0.12$ & 1.03 \\
\hline Enteromorpha sp. & $63.2 \pm 6.6$ & $270.4 \pm 20.3$ & 75 & $2.76 \pm 0.183$ & 1.94 \\
\hline Enteromorpha intestinalis a & $48.3 \pm 6.9$ & $268.6 \pm 39.7$ & 90 & 2.62 & 1.90 \\
\hline
\end{tabular}

$\mathrm{F}$ : frais ; D : dégradé.

${ }^{a}$ Olivier et al. (1996a, b).

Des expériences en milieu contrôlé sur l'appétence que suscitent certains végétaux pour $N$. diversicolor ont montré que cette annélide alloue plus de temps à la recherche de l'algue E. intestinalis qu'aux plantes halophiles fraîches qui appartiennent aux mêmes espèces que celles que nous avons utilisées (Olivier et al., 1995). Cette préférence expliquerait que la croissance des juvéniles $N$. diversicolor de cette présente étude est plus forte avec des détritus frais d'Enteromorpha sp. (taux de croissance pondérale de $1,94 \mathrm{j}^{-1}$ ) que lorsqu'ils sont alimentés avec des détritus frais ou bien des détritus dégradés (Tableau 1). Cette plus forte croissance est en accord avec les résultats obtenus pour d'autres polychètes (Tenore, 1981 ; Olivier et al., 1996a, b). L'interprétation la plus communément invoquée est liée au fait que les algues sont, en règle générale, plus facilement assimilables que les phanérogames marines. En effet, la grande majorité des protéines des algues sont directement disponibles pour les invertébrés benthiques alors que l'utilisation des macromolécules constitutives des tissus de soutien des plantes vasculaires nécessite une dégradation bactérienne (Tenore, 1981).

En conclusion, les résultats de cette étude montrent que les détritus frais de phanérogames halophiles induisent une meilleure croissance des juvéniles de $N$. diversicolor que les détritus dégradés de ces mêmes plantes. Il est vraisemblable que les détritus frais, bien que peu assimilables directement du fait de leur contenu ligno-cellulosique, permettent cette croissance plus élevée en raison de l'ingestion de bactéries et champignons présents probablement en plus grand nombre que sur les détritus dégradés de cette étude.

\section{References}

Findlay, S., Tenore, K.R., 1982. Nitrogen source for a detritivore: detritus substrate versus associated microbes. Science 218, 371-373.
Garça, M.A., Newell, S.Y., Kneib, R.T., 2000. Rates of organic matter and living fungal biomass of decaying Spartina alterniflora. Mar. Biol. 136, 281-289.

Gordon Jr, D.C., Cranford, P.J., Desplanque, C., 1985. Observations on the ecological importance of salt marshes in the Cumberland basin, a macrotidal estuary in the Bay of Fundy. Estuar. Coast. Shelf. Sci. 20, 205-277.

Guillon, L.M., 1984. Les shorres de la baie du Mont Saint-Michel. Unités de végétation et facteurs du milieu. CEE environment, IREC. pp. 78.

Kneib, R.T., Newell, S.Y., Hermeno, E.T., 1997. Survival Growth and reproduction of the salt-marsh amphipod Uhlorchestia spartinophila reared on naturel diets of senescent and dead Spartina alterniflora leaves. Mar. Biol. 128, 423-431.

Lopez, G.R., Levinton, J.S., 1987. Ecology of deposit feeding animals in marine sediments. Q. Rev. Biol. 62, 235-260.

Marty, R., Brenot, S., Retière, C., Desrosiers, G., 1997. First case of adelphophagia studied in Nereidae (Annelida, Polycheta): ecological significance of this behaviour developed by Nereis diversicolor (O.F. Muller). Can. J. Zool. 75, 1575-1584.

Meziane, T., Bodineau, L., Retière, C., Thoumelin, G., 1997. The use of lipid markers to define sources of organic matter in sediment and food web of the intertidal salt marsh-flat ecosystem of Mont Saint-Michel Bay, France. J. Sea Res. 38, 47-58.

Meziane, T., Retière, C., 2001. Role of the biotic interactions on seasonal migrations of the macrozoobenthos living in the upper tidal-flat Mont Saint-Michel Bay, France. Oceanol. Acta 24 (6), 1-7.

Mitsch, W.J., Gosselink, J.G., 1993. Wetlands. Van Nostrand Reinhold, New York

Moran, M.A., Hodson, R.E., 1989. Bacterial secondary production on vascular plant detritus: relationships to detritus composition and degradation rate. Appl. Environ. Microbiol. 55 (9), 2178-2189.

Newel, S.Y., 1993. Decomposition of shoots of a salt marsh grass; methodology and dynamics of microbial assemblages. Adv. Microb. Ecol. 13, 301-326.

Nixon, S.W., 1980. Between coastal marshes and coastal waters - a review of twenty years of speculation and research on the role of salt marshes in estuarine productivity and water chemistry. In: Hamilton, P., McDonalds, K. (Eds.), Estuarine and Wetlands Processes. Plenum Press, New York, pp. 437-535.

Odum, E.P., 1980. The status of three ecosystem-level hypothesis regarding salt marsh estuaries: tidal subsidy, outwelling and detritus based food chain. In: Kennedy, V.S. (Ed.), Estuarine Perspectives. Academic Press, New York, pp. 437-525. 
Olivier, M., Desrosiers, G., Caron, A., Retière, C., Caillou, A., 1995. Behavioral responses of Nereis diversicolor (O.F. Muller) and Nereis virens (Sars) (polychaeta) to food stimuli - use of specific organic matter (algae and halophytes). Can. J. Zool. 73 (12), 2307-2317.

Olivier, M., Desrosiers, G., Caron, A., Retière, C., Caillou, A., 1996. a. Juvenile growth of Nereis diversicolor (O.F. Müller) feeding on a range of marine vascular and macroalgal plant sources under experimental conditions. J. Exp. Mar. Biol. Ecol. 208, 1-12.

Olivier, M., Desrosiers, G., Caron, A., Retière, C., 1996b. Juvenile growth of the polychaete Nereis virens feeding on a range of marine vascular and macroalgal plant sources. Mar. Biol. 125 (4), 693-699.

Rice, D.L., 1982. The detritus nitrogen problem: new observations and perspectives from organic geochemistry. Mar. Ecol. Prog. Ser. 9, $153-162$

Samiaji, J., Bärlocher, F., 1996. Geratology and decomposition of Spartina alterniflora Loisel in a New Brunswick saltmarsh. J. Exp. Mar. Biol. Ecol. 201, 233-252.

Schwinghamer, P., Hargrave, B., Peer, D., Hawkins, C.M., 1986. Partitioning of production and respiration among size groups of organisms in an intertidal benthic community. Mar. Ecol. Prog. Ser. 30, 131-142.

Teal, J.M., 1962. Energy flow in the salt marsh ecosystem of Georgia. Ecology 43, 614-624
Tenore, K.R., 1977. Growth of Capitella capitata cultured on a various levels of detritus derived from different sources. Limnol. Oceanogr. 22, 936-941.

Tenore, K.R., 1981. Organic nitrogen and caloric content of detritus. I. Utilization by the deposit-feeding polychaete Capitella capitata. Estuar. Coast. Shelf Sci. 12, 39-47.

Tenore, K.R., 1983. Organic nitrogen and caloric content of detritus. III. Effects on growth of a deposit-feeding polychaete Capitella capitata. Estuar. Coast. Shelf Sci. 17, 733-742.

Tsuchiya, M., Kurihara, T., 1979. The feeding habits and food sources of the deposit feeding polychaete. Neanthes japonica (Izuka). J. Exp. Mar. Biol. Ecol. 36, 79-89.

Valiela, I., Teal, J.M., 1979. The nitrogen budget of a salt march ecosystem. Nature 280, 655.

Vedel, A., Riisgard, H.U., 1993. Filter-feeding in the polychaete Nereis diversicolor, growth and bioenergetics. Mar. Ecol. Prog. Ser. 100, $145-152$.

Vernberg, F.J., 1993. Salt marsh processes: a review. Environ. Toxicol. Chem. 12, 2167-2195. 